$\xi=$ 离

\title{
Development and evaluation of a customizable electronic medical record for clinical outcomes research and patient engagement
}

\author{
Wayne C. Miller ${ }^{1 *}$, Jennifer Patton ${ }^{2}$, Patricia Lally ${ }^{3}$, Joshua Cusick-Lewis ${ }^{4}$, \\ Karen Fitzpatrick ${ }^{5}$, Haylee Heinsberg ${ }^{2}$ \\ ${ }^{1}$ College of Science, Morehead State University, Morehead, Kentucky, United States of America \\ ${ }^{2}$ West Virginia School of Osteopathic Medicine, Lewisburg, West Virginia, United States of America \\ ${ }^{3}$ Rainelle Medical Center, Rainelle, West Virginia, United States of America \\ ${ }^{4}$ Charleston Area Medical Center, Charleston, West Virginia, United States of America \\ ${ }^{5}$ West Virginia University Family Medicine Center, Morgantown, West Virginia, United States of America \\ *Corresponding author E-mail: w.miller@moreheadstate.edu
}

\begin{abstract}
Background: Electronic Medical Records (EMRs) provide a database to support clinical information needs. However, it is often difficult to access EMR-generated data to answer specific clinical-based questions. Furthermore, EMR is not designed to complete the circle of care by interacting and communicating directly with patients. The problem is even greater in rural practices, with limited resources, and with providers inexperienced in research, who are doing well to meet the daily requirements of keeping their practice doors open.

Objective: Design and evaluate a customizable EMR-Reporting Tool (EMR-RT) that can be used as an adjunct to an existing EMR or as a sole-standing EMR-RT for clinical outcome's research and patient engagement.

Methods: Two rural and two urban family practice clinics participated in the design and beta testing of a customizable EMR-RT for clinical effectiveness research and for patient engagement. The EMR-RT was implemented in each clinic for a 6-month clinical trial.

Results: The EMR-RT used in each clinic was simplistic enough that community health workers could handle patient data entry, data management, and data extraction independently. Each clinic could incorporate clinic-specific measurement variables into the EMR-RT database with minimal effort. Changes to the EMR-RT database capabilities could be performed off-site through the Internet.

Conclusions: A customizable EMR-RT was successfully designed and implemented in two rural and two urban family practice clinics. The EMR-RT was robust enough to congregate clinical research data, but flexible enough and simplistic enough that workers who were previously untrained in EMR use could quickly utilize the system.
\end{abstract}

Keywords: Clinical Research; Electronic Health Record; Electronic Medical Record; Patient Portal.

\section{Introduction}

The development and use of electronic medical records (EMRs) have proliferated exponentially over the past decade. EMRs are recognized as one of the prime transformers of healthcare delivery and central to the success of the patient-centered medical home. From the perspective of patient care, EMRs are expected to improve the accuracy of patient-care information recorded in health records, support clinical decision-making, and improve accessibility of patients' healthcare information for the continuity of care (Zhang and Zhang, 2016). In addition, some EMRs can be used for disease surveillance, management of patient services, and monitoring of patient compliance to treatment plans (Zhang and Zhang, 2016). From a managerial perspective, EMR systems are expected to conserve resources, generate healthcare statistics, and provide meaningful data for the improvement of patient management (Zhang and Zhang, 2016).

Among the myriad of benefits for EMR use, there remain many obstacles to their implementation and/or successful use. Some of these include financial and time constraints associated with implementation, the availability of knowledgeable support personnel, and practitioner frustration with navigating the system (Chang and Gupta, 2015). Other obstacles to EMR acceptance are the inability to search and retrieve information, and difficulties EMRs pose in healthcare communication and coordination (Zhang and Zhang, 2016). Several studies show that the patient-doctor relationship can deteriorate when EMRs prevent doctors from focusing on patients, or when patients cannot see the EMR screen or interact directly with the EMR (Alkureishi, et al., 2016).

The biggest barrier to EMR implementation and use that falls outside of the realm of patient healthcare itself, is the financial burden. Small clinics, single-provider offices, small hospitals, rural healthcare facilities, and many healthcare organizations in developing countries cannot afford the cost of purchasing, operating, and maintaining EMR systems (Chang and Gupta, 2015; Zhang and Zhang, 2016). For many of these entities, there is a lack of business sense to use EMRs. The initial outlay for EMR purchase and the continuing fee-for-service for some EMRs is outright prohibitive (Chang and Gupta, 2015). EMR installation costs range from $\$ 16,000$ to $\$ 36,000$ per physician, with maintenance costs ranging from $\$ 8,000$ to 17,000 per physician per year (Shaha et al., 2015). In order to overcome some of these financial 
barriers to EMR implementation, the use of free and open-source software, without licensing costs, is attractive to those with limited resources who still want the benefits of EMRs (Zhang and Zhang, 2016).

Beyond the financial barriers to purchasing an EMR, there is a growing dissatisfaction among physicians with the EMR platforms available (Shaha et al., 2015). Clinician dissatisfaction with EMRs center around the limitations, inaccessibility, and lack of adaptability imposed by the system. Different fields of medicine, from primary care to specialty areas, have unique requirements for data entry, data extraction, and data analysis. For example, the data requirements for an endocrinologist are different from that of a primary-care physician. However, the EMR requires both physicians to use the same data entry fields, and the extraction and analysis of that data are determined by the EMR software, not the physician. Meaningful use data for a clinician is what he/she needs for continuous improvement in patient care, not for standardized reports dictated by an EMR (Shaha et al., 2015). EMRs need to be adaptable for local clinical needs and programmable in order to maximize the use of relevant patient population data.

Along the lines of EMR adaptability and programmability, comes a series of needs for secondary uses. Clinicians are interested in surveillance of heath events for subsets of their patients. Clinicians are also interested in clinical outcomes research that is specific to their clinical reach and patient population. EMR data must be accessible for ad hoc analysis, and exportable to other analysisfocused systems so that quality-improvement measures can be made to meet the needs of clinicians, patients, and clinical practices (Shaha et al., 2015). With all of this in mind, the objective of this study was to design and evaluate a customizable EMRReporting Tool (EMR-RT) platform that can be used as an adjunct to an existing EMR or as a sole-standing EMR-RT for clinical outcome's research and patient engagement.

\section{Methods}

Two rural and two urban family practice clinics participated in the design and beta testing of a customizable EMR-RT platform for clinical effectiveness research and for patient engagement. Each local community clinic site had its own objectives, depending on how the community clinic site planned to use community health workers (CHWs) to implement the EMR-RT at their respective clinic site. This procedure is an essential part of community-based participatory research - the communities decide what their objective(s) will be and how that objective(s) will be met. In the case of each clinical site, the researchers worked with community partnerships that had a stake in the clinical outcomes - CHWs, community members, clinic administrators, primary care providers, and clinic staff. Once the clinical outcomes were determined for each site, the EMR-RT was developed. The EMR-RT was adapted to capture the desired outcome data for each individual clinical site.

\subsection{Clinical site adaptability}

Rural Clinic 1 (RC1). When necessary, patients from $\mathrm{RC} 1$ are sent to the local hospital. Once a patient is discharged, outpatient treatment is subsequently provided by $\mathrm{RC}$. High-risk patients are those who repeatedly utilize the health care system through multiple emergency-room visits, hospital readmissions, non-adherence to follow-up regimes, and non-adherence to healthy lifestyle behaviors. RC1 used the EMR-RT system to target and track highrisk patients recently discharged from the hospital. The high-risk patients targeted were those admitted for pneumonia, chronic obstructive pulmonary disease, and cardiac episodes (myocardial infarction, atrial fibrillation, or congestive heart failure). The individualized EMR-RT tracked an experimental group of discharged patients and a control group of discharged patients, each of which received different discharge plans. This approach was designed in collaboration with two CHWs, the RC1 staff, the RC1 Chief Executive Officer, the RC1 Medical Director, and researchers; with feedback from patients through case managers. All of those involved in research planning live in the immediate community served. The study parameters were jointly set by these community partners, because that is what they believed met their community's most urgent healthcare needs. Outcome measures tracked for both groups included patient hospital charges, medications, readmissions, emergency-room visits, hospital patient volume, patient vital signs, patient disease awareness, patient behavioral adherence, including lifestyle, and quality of life assessments using the SF-36 Health Status Survey (Ware, 1999). Data were collected through the customized EMR-RT with collaboration between the $\mathrm{RC} 1$ and hospital.

Rural Clinic 2 (RC2). As part of the patient-centered medical home accreditation process, clinics must demonstrate patient selfmanagement activities and tools. RC2 has care plans for patients that contain patient self-derived goals, with some clinician-patientdesigned steps to reach those goals. The study design was where a CHW acted as a Health Coach for patients who had diabetes, hypertension, or obesity. These three diseases were chosen, because they have common lifestyle/behavioral objectives that are woven into the patient-care plans. Patient self-management outcome measures were the number of self-management goals achieved, the rate at which common self-management goals were achieved, participation in prevention behaviors, patient disease awareness, and quality of life assessments using the SF-36 Health Status Survey (Ware, 1999). This approach was designed in collaboration with a $\mathrm{CHW}$, the $\mathrm{RC} 2$ staff, the $\mathrm{RC} 2$ administrators, $\mathrm{RC} 2$ primary care providers, and researchers; with feedback from patients and case managers who worked with patients on self-management. These stakeholders have patient-centered medical home as a top priority, and therefore, decided to help high-risk patients with selfmanagement activities that would help the clinic achieve patientcentered medical home accreditation as well as improve patient outcomes. Other outcomes were hospital admissions, emergencyroom visits, and patient medical profiles. Data for two groups of patients who received two different case management plans were compared, using this customized EMR-RT.

Urban Clinic 1 (UC1). Patients with chronic heart failure, from $\mathrm{UC1}$, were assigned to a CHW to follow the patients after a hospitalization, monitor treatment adherence, refer patients to community resources, and maintain contact with the patient and the UC1. The outcome measures included patient hospital charges, medications, hospital readmissions, emergency-room visits, patient education, patient treatment adherence, and quality of life assessments using the SF-36 Health Status Survey (Ware, 1999). Physicians and staff also developed a checklist that the CHW used to document, and record in the customized EMR-RT, any action taken during their visit with the patient. The treatment arm and standard of care arm of the study differed by post-hospitalization healthcare plan.

Urban Clinic 2 (UC2). Patients from UC2 were assigned to the study based on high emergency department or hospital utilization due to chronic health condition ( 2 or more emergency department visits or hospitalizations within the last year related to chronic health conditions). The UC2 has a well-developed nurse case management program with reporting that enables identification of high utilizer, higher-risk patients. Vulnerable patients are identified by several mechanisms: direct referral by the clinical team to nurse case managers, hospital discharge patient lists, or by registry reports. Outcome measures were taken pre-intervention and at the end of six months. The outcome measures included emergency department and hospital utilization (admissions and readmission), numbers of visits to primary care clinic (including missed and canceled appointments), medications, types of patient education, types of resource referral, and quality of life assessments using the SF-36 Health Status Survey (Ware et al., 1999). 


\section{Results}

Using this EMR-RT for a multi-site clinical trial tested its value as a tool for clinical practice as well as a tool for clinical research. Some of the salient features of this EMR-RT, that were borne out in the research, were:

- Robust - The EMR-RT was robust enough to handle the data from a large patient population. The EMR-RT was structured so that various forms of patient data could be extracted and analyzed for research purposes or for clinical reporting. The robust nature of the database allowed for statistical analyses of data by almost any analytical software (SPSS, for example).

- Flexible - The EMR-RT was flexible in that changes in data collection and data handling could be made at any time. The tool was also flexible enough that modifications in the facepages of the software could be made at any time in order to accommodate clinic or research needs.

- Ubiquitous Use - The EMR-RT was designed to be used in all types of data entry, data extraction, and data analysis. The tool could be used as a supplement to an existing EMR or as a stand-alone EMR. The EMR-RT could be used to send out patient education materials messages to patients via cell phone applications.

- User Friendly - The EMR-RT was friendly enough that community health workers, with no previous EMR experience, learned how to use the tool with a few hours of training. Navigation of the tool was facilitated by the fact that if something was found not to be "user friendly" the software could easily be modified.

- Modifiable - The EMR-RT contained two levels of modification; an end-user ability to modify, and a technology development ability to modify the tool. Both ways of modifying the tool were immediately responsive - meaning modifications could be made quickly, and users did not have to wait for new software versions to be released before they saw changes.

- $\quad$ Remote Access - The EMR-RT could be accessed remotely on two levels. First, the end-user could access the database from any location, and even transport the database, if desired. Second, the software support team could access the database from a remote location to modify or troubleshoot the tool, or to extract data. This capacity allows immediate action to be taken with regard to the database functioning and reporting capacities.

\section{Discussion}

The development and use of EMRs have proliferated exponentially over the past decade. EMRs are recognized as one of the prime transformers of healthcare delivery and central to the success of the patient-centered medical home. The three general benefits EMRs can provide are: solutions to logistical organization problems associated with paper record systems, improvement over the quality of professionals' clinical decisions, and improvement in physicians' return on their practices by reducing the cost of managing clinical information (Pare, et al., 2014). On the other hand, many barriers prevent wider implementation and use of EMRs. Besides being cost-prohibitive, most EMRs are inflexible, not user friendly, inaccessible, and unable to be modified on site (Chang and Gupta, 2015; Shaha et al., 2015; Zhang and Zhang, 2016). The EMR-RT system developed in this study has provided all of the benefits that EMRs are supposed to provide, while overcoming the barriers associated with existing EMRs.

This EMR-RT provides better solutions to organizational problems of paper systems than other EMRs, because this EMR-RT is flexible and can be adapted on site. For example, if a clinician decided to gather information from patients with a survey tool, he/she develops, a traditional EMR cannot incorporate data from that new survey tool into the patient database without significant customization (and thereby time and cost). Thus, the clinician is forced to either use a paper recording system to record the desired data, or use a completely different electronic data capturing tool than that found in the EMR. This process is inefficient and costly. Furthermore, if during a study, a clinician needs to change the data capture by modifying the survey tool or processing the data in a different way, he/she can do that with the EMR-RT, but not with a traditional EMR. With this flexibility and adaptability of the EMR-RT, clinicians make better clinical decisions than with a traditional EMR, because clinicians are not limited to a given set of analyses or reports. The clinicians determine the nature of the analyses and reports themselves, and can easily change these formats, with either on-site EMR-RT adjustments or with the help of remote access technical support. All of this improves the physicians' return on their practices by reducing costs of patient data management.

At the same time, practitioners are gaining the benefits of the EMR-RT, they do not face the barriers associated with traditional EMRs. The EMR-RT is less cost prohibitive than traditional EMRs in that the capital outlay of the EMR-RT is only a fraction of that of traditional EMRs, and there is no continual licensing fee. The EMR-RT is user friendly, in that for the clinical trial reported in this study, community health workers, with no previous training in EMR use, could learn how to use the EMR-RT in only a 3-hour training session. The data capture tools of the EMR-RT are accessible to the end user as well as able to be modified by the end user on site.

\section{Conclusions}

The EMR-RT developed in this study overcomes the major barriers to EMR implementation previously seen, while providing additional benefits for clinical practice. The EMR-RT can be used as a traditional EMR, used as an adjunct to a traditional EMR, or used as a replacement for a traditional EMR. The two greatest assets of the EMR-RT are its low-cost and its adaptability.

\section{Disclaimer}

The EMR-RT developed in this study is not commercially available. It is not the intent of the authors of this study to sell or commercially promote the EMR-RT described throughout this study. The purpose of this study was to show that clinicians and healthcare institutions have other options for EMRs than those existing in the current lucrative marketplace.

\section{Disclosure}

No relevant financial affiliations or conflicts of interest to disclose.

\section{Acknowledgements}

Supported by the West Virginia Clinical and Translation Science Institute, NIH Grant No. 1 U54 GM104942.

\section{References}

[1] Alkureishi MA, Lee WW, Lyons M, Press VG, Imam S, NkansahAmankra A, Werner D, Arora VM. (2016) Impact of electronic medical record use on the patient-doctor relationship and communication: a systematic review. Journal of General Internal Medicine 31(5):548-560. https://doi.org/10.1007/s11606-015-3582-1.

[2] Chang F and Gupta N. (2015) Progress in electronic medical record adoption in Canada. Canadian Family Physician 61:1076-1084.

[3] Zhang X-Y and Zhang P. (2016) Recent perspectives of electronic medical record systems (review). Experimental and Therapeutic Medicine 11:2083-2085. https://doi.org/10.3892/etm.2016.3233. 
[4] Shaha JS, El-Othmani MM, Saleh JK, Bozic, Wright J, Tokish JM, Shaha SH, Saleh KJ. (2015) The growing gap in electronic medical record satisfaction between clinicians and information technology professionals. Journal of Bone and Joint Surgery 97:1979-1984. https://doi.org/10.2106/JBJS.N.01118.

[5] Ware JE Jr. SF-36 Health Survey. Maruish ME (Ed.). The use of psychological testing for treatment planning and outcomes assessment, (2004) $2^{\text {nd }}$ ed., pp 1227-1246. Mahwah, NJ, US: Lawrence Erlbaum Associates Publishers.

[6] Pare G, Raymond L, de Guinea AO, Poba-Nzaou P, Trudel MC Marsan J, and Micheneau T. (2014) Barriers to organizational adoption of EMR systems in family physician practices: a mixedmethods study in Canada. International Journal of Medical Information 83(8):548-558. 